\title{
COVID-19 and the Shift in High Yield Driven Medical Education
}

\author{
Varun Mehta $^{1}$ (D) Sangrag Ganguli ${ }^{1}$
}

Accepted: 3 December 2020 / Published online: 12 January 2021

(C) International Association of Medical Science Educators 2021

\section{To the Editor:}

We read with great interest the article by Vasvada, Ho, and Davison [1]. As medical students, we resonate with the authors' argument that our preclinical education has gained a unique flexibility. The use of deliberate small-group learning to accompany asynchronous lectures has allowed us to retain closeness with our classmates while maintaining the pace of learning new material. In this letter, we further elaborate on a shift in medical education caused by COVID-19: the transition from "high-yield" driven medical education.

Medical education has historically focused on "high yield" concepts. There is a good reason for this: with so much material to learn, it becomes necessary to prioritize the concepts that are important for examinations. Furthermore, as pointed out by Vasvada, Ho, and Davison, many external resources further reinforce these high yield concepts to better prepare us for board examinations [1]. However, as evidenced by this pandemic, a topic deemed "low yield" in the preclinical years can suddenly become very relevant.

The extra time afforded by the flexibility of the online curriculum allowed for a remarkable transformation in medical education amongst our peers: students began to process medicine beyond the "high yield," spending their extra time on interests outside of our classes. Medical students have gotten involved in COVID-19-related research projects, remote volunteering experiences, drive-through testing, and other innovative efforts to combat the pandemic [2].

Additionally, as the bravery of healthcare workers entered the national consciousness, pre-clerkship medical students gained an unparalleled understanding of the risks and benefits of their career path [3]. This has allowed us to stop and reflect

Varun Mehta

varunmehta@gwu.edu

Sangrag Ganguli

sganguli@gwu.edu

1 School of Medicine and Health Sciences, The George Washington University, 2300 I St. NW, Washington, DC 20052, USA rather than just memorize facts. We've asked ourselves questions such as "Do I have the courage to put my life in danger to save others?", "How can I convince others to follow medical protocol despite their personal beliefs?", and "What does medicine mean to me?"

Due to the pandemic, medical students in their preclinical years have withstood the flurry of "high yield" facts while crystallizing their personal goals and motivations in medicine to an unusually mature extent. These observations then prompt an immediate follow-up question, for which there is no easy answer. How can we ensure that such growth and vision are solidified in the early years of medical school for future classes once traditional education resumes?

\section{Compliance with Ethical Standards}

Conflict of Interest The authors declare that they have no conflict of interest.

\section{Ethical Approval NA}

Informed Consent NA

\section{References}

1. Vasavda C, Ho BK, Davison A. Socially distant medical education in the face of COVID-19. Med Sci Educ. 2020;21:1-3. https://doi.org/ 10.1007/s40670-020-01127-4.

2. "How medical students are innovating during COVID-19." American Medical Association, 17 Sept. 2020, www.ama-assn.org/ residents-students/medical-school-life/how-medical-students-areinnovating-during-covid-19.

3. Gallagher TH, Schleyer AM. "We signed up for this!" - student and trainee responses to the Covid-19 pandemic. N Engl J Med. 2020;382(25):e96. https://doi.org/10.1056/NEJMp2005234.

Publisher's Note Springer Nature remains neutral with regard to jurisdictional claims in published maps and institutional affiliations. 